\title{
sciendo
}

\section{The Effectiveness of a Dry-Land Shoulder Rotators Strength Training Program in Injury Prevention in Competitive Swimmers}

\author{
by \\ Nuno Batalha ${ }^{1,2,3}$, Carlos Paixão ${ }^{4}$, António José Silva $a^{3,5,6}$, Mário J. Costa $a^{5,7}$, John \\ Mullen ${ }^{8}$, Tiago M. Barbosa $a^{5,9,10}$
}

\begin{abstract}
Competitive swimmers usually undergo large mileage of daily training, in which propulsive force is produced mainly by the upper limbs. Some studies claim that dry-land shoulder rotators injury prevention programs before the in-water swim practice are paramount. However, the effect of shoulder strengthening prior to water training is unclear. This study aimed to analyse the acute effects of training programs conducted on dry land with the goal of preventing shoulder rotators injuries. A group of young swimmers $(N=23)$ was recruited to participate in this research. The peak torques of shoulder internal and external rotators were assessed before and after the completion of the compensatory strength training program. The isokinetic assessment was performed using two different protocols: 3 repetitions at $60^{\circ} / \mathrm{s}$ and 20 repetitions at 180\%. Except for a trivial reduction in strength after the training program, there were no other significant differences in any of the studied variables (shoulders rotators endurance, strength and muscle balance). All results showed trivial to small effect sizes. Our findings suggest that a compensatory strength training program does not have a significant acute effect on the strength, endurance and muscle balance of shoulder rotators in young swimmers.
\end{abstract}

Key words: swimming, isokinetic strength, shoulder rotators, acute effects.

\section{Introduction}

In competitive swimming, athletes undergo large mileage of daily training, in which 70 percent of the total propelling force is produced by upper limbs. Although propulsive force depends not only on the produced strength, but also on the limbs' orientation (Leong, 2015) and even on how much the fingers are spread (Marinho et al., 2010), it is known that an overload is caused in the shoulder joint. As such, a shoulder rotators imbalance is created, as

reported in competitive swimming (Batalha et al., 2014) and might eventually be related to shoulder injury (Byram et al., 2010) and scapular dyskinesis (Madsen et al., 2011). Swimming requires a large range of motion of the shoulders, with emphasis on circumduction movements that have varying degrees of internal and external rotation and scapular protraction and retraction (Tovin, 2006). The shoulder rotator muscles play a key role in mobility and stability of the glenohumeral joint. A

1 - Departamento de Desporto e Saúde, Escola de Ciência e Tecnologia, Universidade de Évora, Évora, Portugal.

2 - CHRC-UE - Comprehensive Health Research Center, University of Évora, Portugal.

3 - Federação Portuguesa de Natação,Lisboa, Portugal.

4 - Sub-Dep. de Desporto, Escola Superior de Educação, Instituto Politécnico de Beja, Portugal.

5 - Research Centre of Sports, Health and Human Development, CIDESD, STRONG Research Community. Portugal.

6 - University of Trás-os-Montes and Alto Douro. Vila Real - Portugal.

7 - Sports Science Department, Polytechnic Institute of Guarda, Portugal.

8 - COR Physical Therapy and Sports Performance, Santa Clara, California. United States.

9 - National Institute of Education, Nanyang Technological University, Singapore.

10 - Department of Sport Sciences, Polytechnic Institute of Bragança, Bragança, Portugal. 
slight imbalance in the relationship between the internal rotators (IR) and the external rotators (ER) of the shoulder, may result in dysfunctions or joint damage (Batalha et al., 2013). Shoulder muscle imbalances indicated by low values of the ER/IR ratio, have been observed in individuals with glenohumeral joint instability, and are considered a shoulder injury risk factor (Lin, 2015; Niederbracht et al., 2008). It has previously been shown that in swimming, an in-water training macrocycle is enough to trigger muscle imbalances in swimmer's shoulder rotators (Batalha et al., 2014). This is due to a significant increase in IR strength, with no concurrent increase in ER strength (Batalha et al., 2014). Hence, it seems necessary to design and implement programs with the goal to prevent shoulders' injuries.

The intervention programs proposed earlier (Leppänen, 2014; Parkkari, 2001) are based on longitudinal designs, reporting their effectiveness. In a recent study, Batalha et al. (2015) noted that a compensatory strength training program had beneficial effects on the rotator cuff muscles, providing not only increased strength values of ER and IR, but also increasing muscular balance between them. In the same way, Wanivenhaus et al. (2012), showed that a complete program with shoulder flexibility and strengthening routines should be part of a training program in competitive swimming. Complementarily, Gaunt and Maffulli (2012) noted that a shoulder strengthening program must include a high number of repetitions and focus also on muscular endurance capacity that is required when swimming.

Referring to studies on shoulder injury prevention training conducted with swimmers (Batalha et al., 2014; Kluemper et al., 2006; Stanula et al., 2012; Van de Velde et al., 2011) we can note that training programs are usually carried out before the in-water swim session. This approach raises questions if it might elicit fatigue over the strength training session and have an impact on the in-water session. Coaches do have concerns about strength and conditioning sessions taking place before the in-water session. The rationale is that swimmers will already be exhausted after the dry-land program, which could affect their inwater performance. To our knowledge, no prior study has attempted to address this question.
Therefore, the aim of this study was to evaluate the acute effects of an injury prevention training program for the shoulder rotator cuff. It was hypothesised that such a program would promote a decrease in shoulder rotators balance (lower strength ratios), strength and endurance.

\section{Methods}

\section{Participants}

A group of 23 swimmers (Table 1), all competing at the national level, were recruited for this research. Of the total sample, $60.87 \%(n=14)$ were males and $39.13 \%(n=9)$ were females. Only two swimmers had their left arm as the dominant upper limb.

As inclusion criteria, swimmers should: i) not have any clinical history of upper limb disorders; ii) be aged 15 years-old and above; iii) undergo a minimum of 8 hours of training per week.

This study was approved by the Institutional Review Board of the hosting university (process: GD/40477/2014/P1) and was undertaken in compliance with the Helsinki Declaration and the international principles governing research on humans and animals. Participants and their legal guardians were briefed on the aims and procedures. Written consent forms were signed by both participants and legal guardians.

\section{Procedures}

Figure 1 portrays the selected experimental design. Two evaluation moments were selected to assess the shoulder rotators strength. The first evaluation was prior to the compensatory strength training program and the second immediately after the completion of the program, without any recovery time. The first evaluation was always preceded by a $10-\mathrm{min}$ warm-up for swimmers to perform a general upper limb joint mobilization and stretching program. After completion of the first moment of evaluation and prior to the compensatory strength training program, there was a minimum of $15 \mathrm{~min}$ of recovery.

All isokinetic IR and ER shoulder strength values were evaluated on a dynamometer (Biodex System 3, Biodex Corp., Shirley, NY, USA), properly calibrated. Each participant reported to the laboratory one week prior to testing for familiarization with equipment and procedures. 
One week after familiarization, swimmers' sokinetic internal and external concentric shoulder strength was evaluated in the sitting position, with the arm at $90^{\circ}$ of abduction in the sagittal plane and elbow flexion at $90^{\circ}$. Participants were seated and stabilized using velcro straps to avoid compensatory trunk movements. We also defined the neutral position with the arm at $90^{\circ}$ of abduction and elbow flexion, as the initial position. Participants began testing in the previously described position, performing a range of motion from $90^{\circ}$, from neutral rotation to $90^{\circ}$ of external rotation.

The correction to the effect of gravity, with the relaxed arm in the neutral starting position and alignment of joints (the shoulder joint center aligned with the dynamometer axis) was performed in all the assessments carried out in accordance to Biodex's recommendations.

Regarding the evaluation protocol, we chose to carry out the same that was reported in the literature, namely in Heinlein and Cosgarea (2010), Wanivenhaus et al. (2012): (i) Protocol 1 performing 3 repetitions at an angular velocity of 60\% $/ \mathrm{s}$; (ii) Protocol 2 - performing 20 repetitions at an angular velocity of $180 \%$ s. Verbal encouragement provided by the evaluator during performance of both protocols was constant.

An explanation of the testing procedure was given to all participants before the test, with an emphasis on having each athlete exerting maximal effort within his/her tolerance level. Three trial repetitions at each speed were performed to familiarize participants with the testing procedure. The $60 \%$ speed was first performed for each limb, followed up by the $180^{\circ} / \mathrm{s}$ speed. A two-minute rest interval was allowed between each speed test. The limbs' order of testing was randomly assigned.

To monitor the shoulder rotator strength, peak torque (PT) was used. PT was defined as the maximum torque produced by the shoulder at any point in the range of motion (Perrin, 1993). To analyse shoulder rotator strength balance, the ER/IR ratio [the ratio between PT values of ER and IR: (ER-PT/IR-PT) x 100] was calculated. The definition of the Fatigue Index (FI) was performed according to the manufacturer's recommendations (Biodex Corporation, 1995), as follows [(W1 - W2)/W1] x 100, where W1 and W2 represent the mechanical work of the first third and last third of the repetitions, respectively. This variable, being an indicator of muscle fatigue, was only used by the protocol with 20 repetitions at $180^{\circ} \cdot \mathrm{s}^{1}$.

The shoulder compensatory strength training program was performed after a minimum recovery period of 15 minutes that followed the first evaluation.

Based on the literature, the compensatory strength training program was designed with exercises that present a moderate to high electromyographic activation level of the rotator cuff muscles (Dark et al., 2007; Escamilla et al., 2009). The program was performed using resistance bands (Thera-Band $\left.{ }^{\circledR}\right)$, which are dryland training devices used by swimmers on regular basis and considered effective at developing muscle endurance (Andersen et al., 2010). The training program consisted of 4 different exercises (Figure 2). All swimmers performed three sets of 20 repetitions of each exercise with $30 \mathrm{~s}$ of rest intervals between repetitions and one minute between exercises. We used a high number of repetitions of exercises to meet the specificity of swimming, which requires muscular endurance capacity and to obtain a larger solicitation by rotator cuff muscles (Gaunt and Maffulli, 2012). The correct technical execution of the exercise by the swimmers was a constant concern. It should be noted that it was always the same researcher who monitored and supervised the training sessions. All participants started the exercises with the resistance band in a neutral length, under minimum tension.

Exercise 1 - Shoulder press: initial position upright, placing the shoulder in $90^{\circ}$ of flexion in the scapular plane, the elbow in full flexion and pronation hands above the shoulders. Then, elbows perform a full pronation and a full shoulder flexion, slowly returning to the initial position.

- Exercise 2 - Shoulder external rotation with the arm abducted to $90^{\circ}$ : initial position upright, with shoulders' $90^{\circ}$ abduction and elbows at $90^{\circ}$ of flexion. Then, the shoulder performs an external rotation until the hand is aligned with the head, slowly returning to the initial position.

- Exercise 3 - Shoulder flexion above $120^{\circ}$ in external rotation (thumbs up): initial position like the anatomical reference position. Then, the 
arms perform simultaneously a flexion above $120^{\circ}$ and slowly return to the initial position.

- Exercise 4 - Low scapular rows at $45^{\circ}$ of flexion: initial position upright, with the elbows at $45^{\circ}$ deflection. Then, the elbows perform a low scapular row movement, slowly returning to the initial position.

The Thera-Band ${ }^{\circledR}$ Manual claims that the blue, black, silver and gold bands are typically used by athletes. Thus, to determine the initial training load, after two sessions of adaptation to the material and performance technique, swimmers performed the test with blue (female participants) and black (male participants) elastic bands. The test consisted of performing two sets of 20 repetitions and one last set to exhaustion. Swimmers who completed the last set of 30 repetitions with appropriate technique, started training with a band of higher resistance. This test was valid for each of the exercises independently, having already been used in previous studies (Batalha et al., 2015).

\section{Statistical analysis}

All analyses were performed using SPSS (version 22.0; SPSS, Inc., Chicago, IL, USA), and the significance level was set at $p \leq .050$ for all tests. Initially, the Shapiro-Wilk normality test with Lilliefors correction was used to assess data normality. To compare the values before and after the training program, a Student's t-test for paired samples was selected. Concurrently to null hypothesis testing, the mean and $95 \%$ confidence interval were also computed, to best characterise changes over time. Effect sizes were also calculated (Cohen's d) being assessed as trivial (0$0.19)$, small (0.20-0.49), medium (0.50-0.79) and large (>0.80) (Cohen, 1988).

\section{Results}

Except for a trivial decrease in the strength values of ER and IR after the completion of training, there were no other significant differences between pre- and post-assessment, considering the dominant shoulder (DS) and exercise at the angular velocity of $60 \%$ s. Consequently, there were also no differences between the ER/IR ratio values (Table 2).

For the same angular velocity, but for the non-dominant shoulder (NDS), results were similar (Table 2). No differences with trivial effect sizes were observed after the compensatory strength training program in all variables, ER-PT $(p=.334 ; d=-.109)$, IR-PT $(p=.902 ; d=.014)$ and ER/IR ratios $(p=.369 ; d=-.018)$.

Table 3 depicts the results at $180^{\circ} / \mathrm{s}$. We can note that also in this protocol, the compensatory strength training program did not induce any significant acute effect in the same variables (ER-PT: $p=.264 ; d=-.146$; IR-PT: $p=.138$; $d=-.211$; ER/IR ratios: $p=.750 ; d=.070$ ). Small effect sizes and non-significant differences were also observed in ER-FI $(p=.910 ; d=.017)$ and IR-FI $(p=.102 ; d=.320)$.

For the NDS, at the same angular velocity $\left(180^{\circ} / \mathrm{s}\right)$, results were similar to what was reported for DS. Small effect sizes and non-significant differences were noted for the same variables, meaning that the compensatory strength training program did not induce significant fatigue.

\section{Table 1}

Sample $(n=23)$ characteristics (mean \pm standard deviation $(S D)$ )

\begin{tabular}{cc}
\hline & Mean \pm SD \\
\hline Age (years) & $16.43 \pm 1.38$ \\
Body height $(\mathrm{cm})$ & $168.61 \pm 7.91$ \\
Body mass $(\mathrm{kg})$ & $58.97 \pm 7.75$ \\
BMI $\left(\mathrm{kg} / \mathrm{m}^{2}\right)$ & $20.76 \pm 2.54$ \\
\hline
\end{tabular}




\section{Table 2}

Acute effects of the injury prevention program on IR and ER Peak-Torques (Nm) and $E R / I R$ ratios (\%) of both shoulders at $60^{\circ} / \mathrm{s}$.

\begin{tabular}{|c|c|c|c|c|c|}
\hline \multicolumn{6}{|c|}{ Dominant shoulder $-60 \% / \mathrm{s}$} \\
\hline & $\begin{array}{l}\text { Pre-intervention } \\
(\text { Mean } \pm \text { SD) }\end{array}$ & $\begin{array}{l}\text { Post-intervention } \\
(\text { Mean } \pm \text { SD })\end{array}$ & $\begin{array}{c}\text { Difference } \\
\text { Mean }(95 \% \text { CI })\end{array}$ & $p$ & ES \\
\hline ER-PT & $24.29 \pm 7.96$ & $24.09 \pm 7.40$ & $-0.20(-1.66$ to 2.06$)$ & .826 & -.026 \\
\hline IR-PT & $34.57 \pm 12.09$ & $32.27 \pm 10.49$ & $-2.30(-0.23$ to 4.83$)$ & .072 & -.203 \\
\hline ER/IR ratio & $73.50 \pm 18.91$ & $77.37 \pm 16.40$ & $3.87(-11.17$ to 7.43$)$ & .283 & .218 \\
\hline \multicolumn{6}{|c|}{ Non-dominant shoulder $-60 \% \mathrm{~s}$} \\
\hline & $\begin{array}{l}\text { Pre-intervention } \\
(\text { Mean } \pm \text { SD) }\end{array}$ & $\begin{array}{l}\text { Post-intervention } \\
(\text { Mean } \pm \text { SD })\end{array}$ & $\begin{array}{c}\text { Difference } \\
\text { Mean }(95 \% \text { CI })\end{array}$ & $p$ & ES \\
\hline ER-PT & $23.72 \pm 7.46$ & $22.96 \pm 6.36$ & $-0.76(-0.84$ to 2.35$)$ & .334 & -.109 \\
\hline IR-PT & $32.67 \pm 10.68$ & $32.82 \pm 9.37$ & 0.14 (-2.50 to 2.22$)$ & .902 & .014 \\
\hline ER/IR ratio & $75.34 \pm 17.08$ & $72.31 \pm 15.66$ & $-3.03(-3.82$ to 9.88$)$ & .369 & -.018 \\
\hline
\end{tabular}

$p-t$-student test for paired samples; ES - Cohen's d effect sizes

Table 3

Acute effects of the injury prevention program on IR and ER Peak-Torques (Nm) and $E R / I R$ ratios (\%) of both shoulders at $180^{\circ} \cdot s^{-1}$.

\begin{tabular}{|c|c|c|c|c|c|}
\hline \multicolumn{6}{|c|}{ Dominant shoulder $-180^{\circ} / \mathrm{s}$} \\
\hline & $\begin{array}{l}\text { Pre-intervention } \\
\quad(\text { Mean } \pm \text { SD })\end{array}$ & $\begin{array}{l}\text { Post-intervention } \\
(\text { Mean } \pm \text { SD) }\end{array}$ & $\begin{array}{c}\text { Difference } \\
\text { Mean }(95 \% \text { CI })\end{array}$ & $p$ & ES \\
\hline ER-PT & $23.40 \pm 6.07$ & $22.54 \pm 5.70$ & $-0.86(-1.70$ to 2.42$)$ & 264 & - - 146 \\
\hline IR-PT & $33.04 \pm 12.24$ & $30.71 \pm 9.67$ & $-2.33(-0.81$ to 5.47$)$ & .138 & -.211 \\
\hline ER/IR ratio & $75.52 \pm 18.54$ & $76.76 \pm 16.38$ & $1.24(-9.21$ to 6.73$)$ & .750 & .070 \\
\hline ER-FI & $29.80 \pm 10.84$ & $29.57 \pm 14.91$ & $-0.23(-3.87$ to 4.32$)$ & .910 & -.017 \\
\hline IR-FI & $21.48 \pm 10.67$ & $24.81 \pm 10.14$ & 3.33 (-5.38 to 4.72$)$ & .102 & .320 \\
\hline \multicolumn{6}{|c|}{ Non-dominant shoulder $-180 \% / \mathrm{s}$} \\
\hline & $\begin{array}{l}\text { Pre-intervention } \\
\quad(\text { Mean } \pm \text { SD })\end{array}$ & $\begin{array}{l}\text { Post-intervention } \\
\quad(\text { Mean } \pm \text { SD) }\end{array}$ & $\begin{array}{c}\text { Difference } \\
\text { Mean }(95 \% \text { CI) }\end{array}$ & $p$ & ES \\
\hline ER-PT & $22.23 \pm 6.44$ & $21.97 \pm 5.22$ & $-0.26(-1.09$ to 1.62$)$ & .688 & -.044 \\
\hline IR-PT & $31.71 \pm 9.93$ & $30.94 \pm 9.92$ & $-0.77(-1.68$ to 3.21$)$ & .522 & -.077 \\
\hline ER/IR ratio & $71.43 \pm 12.29$ & $74.28 \pm 13.99$ & 2.86 (-8.77 to 3.06$)$ & .327 & .216 \\
\hline ER-FI & $29.25 \pm 8.76$ & $31.69 \pm 14.67$ & $2.44(-9.24$ to 4.35$)$ & .464 & .202 \\
\hline IR-FI & $18.77 \pm 11.88$ & $21.65 \pm 14.57$ & 2.87 (-8.60 to 2.85$)$ & .309 & .217 \\
\hline
\end{tabular}

$p-t$-student test for paired samples; ES - Cohen's d effect sizes 


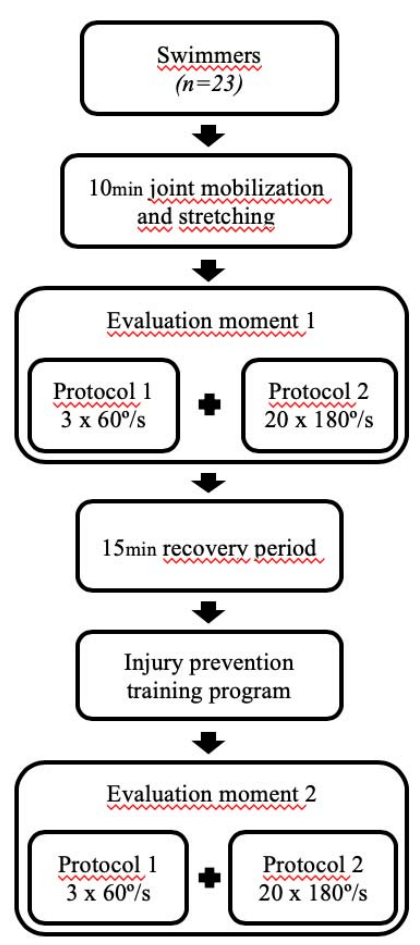

Figure 1

Study design
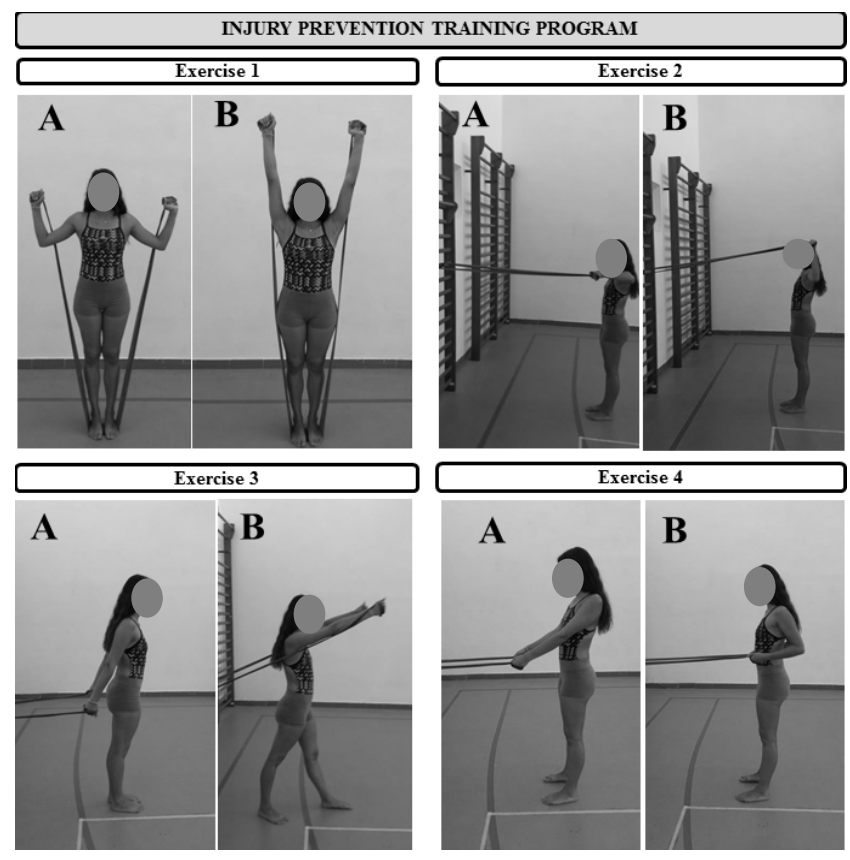

Figure 2

Injury prevention training program ( $A$ - Initial position; $B$ - Final position) 


\section{Discussion}

The aim of this study was to evaluate the acute effects of a shoulder rotators injury prevention training program in swimmers. The results did not confirm the hypothesis under testing. Such a training program does not seem to cause a significant change in shoulder rotators strength, endurance and muscle balance.

The IR and ER strength results of our study are in agreement with literature (Batalha et al., 2015; Ellenbecker and Roetert, 2003; Gozlan et al., 2006). A superior ability of the IR to produce strength compared to their antagonists was reported in both protocols (60\% $/ \mathrm{s}$ and $\left.180^{\circ} / \mathrm{s}\right)$ and in the two shoulders. This is sustained with the fact that the IRs are more stimulated in the swimming actions, and consequently stronger (Dark et al., 2007; Ellenbecker and Roetert, 2003).

Regarding the internal and external rotators PT values, there were non-significant differences before and after the strength training program in both protocols. Apart from the IR PT of the NDS at $60^{\circ} / \mathrm{s}$, which increased by $0.14 \mathrm{Nm}$ $(\mathrm{p}=.902 ; \mathrm{d}=.014)$, all values presented a slight decrease after the training program. Our ER and IR strength values (from 23.40 to $34.57 \mathrm{Nm}$ in the DS; and from 22.23 to $32.67 \mathrm{Nm}$ in the NDS) are very similar to those reported in other studies that also recruited age-group swimmers (Batalha et al., 2013; Batalha et al., 2014). However, Olivier et al. (2008) reported PT of ER and IR from 28.7 to 61.2 $\mathrm{Nm}$ in the DS; and from 27.8 to $52.8 \mathrm{Nm}$ in the NDS. These ranges are significantly wider than those found in our study. However, the latter study recruited high-level swimmers with a mean age of 29 years-old and not age-group swimmers. On the other hand, we consider that it may be important to transfer this study data to other populations, namely a high-level sample and, consequently, an older one. This is justified by the fact that we may have different results compared to studies with older and qualitatively better samples, with different strength results (Olivier et al., 2008) and ratios (Gozlan et al., 2006).

Previous studies have reported that the ER/IR ratio assessment can be a useful measure to identify muscle imbalances in the swimmers' shoulders, being also associated with possible injuries (Ellenbecker and Davies, 2000). Previous normative data deemed as adequate ER/IR ratios between 66 and 75\% (Cingel et al., 2007;
Ellenbecker and Davies, 2000). However, not all studies recruited swimmers. Values below this threshold are commonly associated to instability and muscle imbalance in the glenohumeral joint (Ellenbecker and Roetert, 2003), with higher risk of injury in the pre-season (Byram et al., 2010).

The ratio values obtained in the first assessment of our study ranged between $71.43 \pm$ $2.29 \%$ and $75.52 \pm 18.54 \%$ in both protocols (Tables 2 and 3). Therefore, these values are within the reference values provided by literature for muscular balance of the shoulder rotators. After the strength training program, changes in the ER/IR ratios did not follow a uniform pattern, depending on the protocol used and the evaluated limb. Thus, in the protocol of $60 \%$ s, the DS ER/IR ratio increased by $3.87 \pm 16.87 \%$, to a value of $77.37 \pm 16.40 \%$. In the same protocol, but for the NDS, we obtained a decrease in the ER/IR ratio ($3.03 \pm 15.84 \%$ ) (Table 2). In a previous study, recruiting young swimmers, Batalha et al. (2013) reported similar ratios, also higher than $70 \%$ $(79.39 \pm 15.4 \%$ for the DS and $74.56 \pm 18.66 \%$ for the NDS). On the other hand, Gozlan et al. (2006) reported lower ratios in a sample of 17.2 year-old swimmers. In the $60 \%$ s protocol, the results varied between 39.77 and $45.36 \%$.

Considering the $180^{\circ}$ /s protocol, the ER/IR ratio values in both shoulders slightly increased (DS $=1.24 \pm 18.43 \%$, to a value of $76.76 \pm 16.38 \%$ and NDS $=2.86 \pm 13.68 \%$ to a value of $74.28 \pm$ $13.99 \%$ ) (Table 3 ). In this protocol, Batalha et al. (2013) obtained ratios for DS $=77.04 \pm 12.99 \%$ and for NDS $=73.83 \pm 13.68 \%$, values similar to those from our study. Once again, Gozlan et al. (2006) obtained lower ratios in the $180 \%$ s protocol. Those authors noted values between 38.04 and $44.19 \%$. In that study, however, the evaluation position of the arm was $45^{\circ}$ of abduction, which could explain the differences. In a study with older swimmers (29 \pm 5 years old), Olivier et al. (2008) obtained similar values in the $180^{\circ}$ /s protocol only (DS $=71.4 \%$ and NDS $=72.5 \%$ ). In the $60 \% / \mathrm{s}$ protocol, those authors noted lower values, even below the reference guidelines (DS $=53.27 \%$ and NDS $=65.90 \%$ ). However, they assessed participants in the supine position, which may also justify the difference. It is well known that isokinetic strength is widely recognized. However, the position chosen to perform the evaluation process may influence the results 
(Radaelli et al., 2010). We acknowledge that the prone position may have been more suitable for our sample; however, this option was not available in our dynamometer.

Although there is still some controversy among researchers as to whether absolute strength or strength ratios should be used to quantify optimal levels of dynamic shoulder stability (Leroux et al., 1994), there is a degree of consensus regarding unilateral ratios as one of the most important variables to be characterized when seeking to diagnose the muscular balance/imbalance of a given joint (Ellenbecker and Roetert, 2003). In this sense, and based on the normative unilateral ratios data, we can confirm that in all evaluations no values worthy of concern were found (ER/IR ratios did not register values below $66 \%$ at any point), which according to Leroux et al. (1994), are not associated with severe imbalances. In short, conducting an injury prevention strength training program seems to have no effect on the shoulder rotators muscle balance.

In addition to the analysis of muscle strength and balance, it would be interesting to verify the training effect on muscle endurance, which is important in swimming. In this sense, the FI was evaluated. In the IR of the DS, there was an increase of $3.33 \pm 9.37 \%$ (Table 3), however, with non-significant differences. The ER had a different behaviour, decreasing slightly $(0.23 \pm 9.47 \%)$. In the NDS, there were also nonsignificant differences in both shoulder rotators. Thus, our results reject the hypothesis that a strength program would reduce the muscle endurance of shoulder rotators.

It is also worth noting the results of the evaluations carried out in both groups. The value of the IR-FI was always lower than the value of the ER-FI, specifically, the ER had lower fatigue resistance than the IR. This difference may be related to the fact that the IR have a higher power generation capacity relative to the ER, as they are anatomically larger and in the greater number (Batalha et al., 2015; Dark et al., 2007; Ellenbecker and Roetert, 2003).

There are limited data on the FI. With a shortage of studies analysing this variable, it made it difficult to achieve a theoretical framework of the obtained data. However, in the study of Batalha et al. (2013), the FI was also collected. Those authors reported DS values of $14.19 \pm 8.54 \%$ in the ER and $5.94 \pm 2.73 \%$ in the IR. For the NDS, the FI was $15.76 \pm 6.74 \%$ for the ER and $5.96 \pm 3.14 \%$ for the IR. These values were low, differing significantly from the ones registered in our study. However, swimmers in the referred study (Batalha et al., 2013) performed 32 weeks of a training program, which may explain the differences.

We acknowledge a few limitations of our study. The main one concerns the performed isokinetic test, in which participants were in a position non-specific for swimming. A prone testing position may be more suitable for swimmers; however, this option was not available in our dynamometer. We also consider that the sample size and gender gap were limitations. In future studies, the sample should be larger and possible gender differences should be explored.

\section{Conclusions}

Based on our results, shoulder rotators strength, endurance and balance do not seem to be impaired after undergoing a shoulder rotators injury prevention training program. Thus, and as a practical implication, it seems appropriate that coaches can design and implement strength exercises for shoulder muscle reinforcement, prior to in-water swim sessions.

\section{References}

Andersen LL, Andersen CH, Mortensen OS, Poulsen OM, Bjornlund IB, Zebis MK. Muscle activation and perceived loading during rehabilitation exercises: comparison of dumbbells and elastic resistance. Phys Ther, 2010; 90: 538-549

Batalha N, Marmeleira J, Garrido N, Silva AJ. Does a water-training macrocycle really create imbalances in swimmers' shoulder rotator muscles? Eur J Sport Sci, 2015; 15: 167-172

Batalha N, Raimundo AM, Tomas-Carus P, Barbosa TM, Silva AJ. Shoulder rotator cuff balance, strength, and endurance in young swimmers during a competitive season. J Strength Cond Res, 2013; 27: 25622568 
Batalha N, Raimundo AM, Tomas-Carus P, Marques MA, Silva AJ. Does an in-season detraining period affect the shoulder rotator cuff strength and balance of young swimmers? J Strength Cond Res, 2014; 28: 2054-2062

Byram IR, Bushnell BD, Dugger K, Charron K, Harrell FE, Jr., Noonan TJ. Preseason shoulder strength measurements in professional baseball pitchers: identifying players at risk for injury. Am J Sports Med, 2010; 38: 1375-1382

Cingel R, Kleinrensinkb G, Mulderc P, Bied R, Kuiperse H. Isokinetic strength values, conventional ratio and dynamic control ratio of shoulder rotator muscles in elite badminton players. Isokinet Exerc Sci, 2007; 15: 287-93

Cohen J. Statistical power analysis for the behavioral sciences $2 n d$ ed. Hillsdale, NJ: Lawrence Erlbaum; 1988

Dark A, Ginn KA, Halaki M. Shoulder muscle recruitment patterns during commonly used rotator cuff exercises: an electromyographic study. Phys Ther, 2007; 87: 1039-1046

Ellenbecker TS, Davies GJ. The application of isokinetics in testing and rehabilitation of the shoulder complex. J Athl Train, 2000; 35: 338-350

Ellenbecker TS, Roetert EP. Age specific isokinetic glenohumeral internal and external rotation strength in elite junior tennis players. J Sci Med Sport, 2003; 6: 63-70

Escamilla RF, Yamashiro K, Paulos L, Andrews JR. Shoulder muscle activity and function in common shoulder rehabilitation exercises. Sports Med, 2009; 39: 663-685

Gaunt T, Maffulli N. Soothing suffering swimmers: a systematic review of the epidemiology, diagnosis, treatment and rehabilitation of musculoskeletal injuries in competitive swimmers. Br Med Bull, 2012; 103: $45-88$

Gozlan G, Bensoussan L, Coudreuse JM, Fondarai J, Gremeaux V, Viton JM, Delarque A. Isokinetic dynamometer measurement of shoulder rotational strength in healthy elite athletes (swimming, volley-ball, tennis): comparison between dominant and nondominant shoulder. Ann Readapt Med Phys, 2006; 49: 8-15

Heinlein SA, Cosgarea AJ. Biomechanical Considerations in the Competitive Swimmer's Shoulder. Sports Health, 2010; 2: 519-525

Kluemper M, Uhl T, Hazelrigg H. Effect of stretching and strengthening shoulder muscles on forward shoulder posture in competitive swimmers. J Sport Rehabil, 2006; 15: 58-70

Leong, J. (2015). The fundamentals behind curvilinear vs straight line pull. Journal of Swimming Research, 23, $14-20$

Leppänen M. Interventions to Prevent Sports Related Injuries: A Systematic Review and Meta-Analysis of Randomised Controlled Trials. Sports Med, 2014; 44: 473-486

Leroux JL, Codine P, Thomas E, Pocholle M, Mailhe D, Blotman F. Isokinetic evaluation of rotational strength in normal shoulders and shoulders with impingement syndrome. Clin Orthop Relat Res, 1994; 304: 108-115

Lin HT. The changes in shoulder rotation strength ratio for various shoulder positions and speeds in the scapular plane between baseball players and non-players. J Phys Ther Sci, 2015;5(27):1559-1563

Madsen PH, Bak K, Jensen S, Welter U. Training induces scapular dyskinesis in pain-free competitive swimmers: a reliability and observational study. Clin J Sport Med, 2011; 21: 109-13

Marinho DA, Barbosa TM, Reis VM, Kjendlie PL, Alves FB, Vilas-Boas JP, Machado L, Silva AJ, Rouboa AI. Swimming propulsion forces are enhanced by a small finger spread. J Appl Biomech, 2010; 26: 87-92

Niederbracht Y, Shim AL, Sloniger MA, Paternostro-Bayles M, Short TH. Effects of a shoulder injury prevention strength training program on eccentric external rotator muscle strength and glenohumeral joint imbalance in female overhead activity athletes. J Strength Cond Res, 2008; 22: 140-145

Olivier N, Quintin G, Rogez J. The high-level swimmer articular shoulder complex. Ann Readapt Med Phys, 2008; 51: 342-347 
Parkkari J. Is it Possible to Prevent Sports Injuries? Review of Controlled Clinical Trials and Recommendations for Future Work. Sports Med. 2001; 31: 985-995

Perrin DH. Isokinetic Exercise and Assessment. Kinetics H, editor. Champaign, IL1993

Tovin BJ. Prevention and Treatment of Swimmer's Shoulder. N Am J Sports Phys Ther, 2006; 1:166-175

Radaelli R, Bottaro M, Weber F, Brown L, Pinto R. Influence of body position on shoulder rotator muscle strength during isokinetic assessment. Isokinet Exerc Sci, 2010; 18(3): 119-124

Stanula A., Maszczyk A, Roczniok R, Pietraszewski P, Ostrowski A, Zając A, Strzała M. The Development and Prediction of Athletic Performance in Freestyle Swimming. J Hum Kinet, 2012; 32: 97-107

Van de Velde A, De Mey K, Maenhout A, Calders P, Cools AM. Scapular-muscle performance: two training programs in adolescent swimmers. J Athl Train. 2011; 46: 160-167

Wanivenhaus F, Fox AJ, Chaudhury S, Rodeo SA. Epidemiology of injuries and prevention strategies in competitive swimmers. Sports Health. 2012; 4: 246-251

\section{Corresponding author:}

\section{Nuno Batalha}

Adress: University of Évora. Sport and Health Department.

R. Reguengos de Monsaraz, no 14 . 7000-727 Évora - Portugal.

Phone/Fax: (+351)962435000 - (+351)266769521

E-mail: nmpba@uevora.pt 